\title{
On a study of the exponential and Poisson characteristics of the Poisson process*
}

\author{
Wen-Jang Huang, Wen-Ching Chang \\ Department of Applied Mathematics, National Sun Yat-sen University, Kaohsiung, Taiwan, \\ 80424, R.O.C. (e-mail: huangwj@math.nsysu.edu.tw)
}

Received: August 1999

\begin{abstract}
Based on the exponential and Poisson characteristics of the Poisson process, in this work we present some characterizations of the Poisson process as a renewal process. More precisely, let $\gamma_{t}$ be the residual life at time $t$ of the renewal process $A=\{A(t), t \geq 0\}$, under suitable condition, we prove that if $\operatorname{Var}\left(\gamma_{t}\right)=E^{2}\left(\gamma_{t}\right), \forall t \geq 0$, then $A$ is a Poisson process. Secondly, we show that if $\operatorname{Var}(A(t))$ is proportional to $E(A(t))$, then $A$ is a Poisson process also, and $\operatorname{Var}(A(t))=E(A(t))$.
\end{abstract}

Key words: Characterization, exponential distribution, Poisson distribution, Poisson process, renewal process

\section{Introduction}

In this work, we will investigate what kind of conditions are necessary to force a renewal process to become a Poisson process. Let $\left\{X_{n}, n \geq 1\right\}$ be a sequence of independent and identically distributed (i.i.d.) random variables with common distribution function $F$. Throughtout this paper we assume $F(0)=0$. Let $S_{0}=0, S_{n}=\sum_{i=1}^{n} X_{i}, n \geq 1$. Also let $A(t)=\sup \left\{n \mid S_{n} \leq t\right\}, t \geq 0$, denote the number of renewals in $[0, t]$. The random variables $\delta_{t}=t-S_{A(t)}, \gamma_{t}=$ $S_{A(t)+1}-t$, and $\beta_{t}=S_{A(t)+1}-S_{A(t)}$, will be called respectively "current life at $t$ ", "residual life at $t$ ", and "total life at $t$ " of the (ordinary) renewal process $A=\{A(t), t \geq 0\}$. In the above set up, if $\left\{X_{n}, n \geq 1\right\}$ are still assumed to be independent, but only $X_{2}, X_{3}, \ldots$ are identically distributed with the common distribution function $F$, while $X_{1}$ has possibly a different distribution function, say $H$ with $H(0)=0$, then $\left\{X_{n}, n \geq 1\right\}$ form the interarrival times of

\footnotetext{
* Support for this research was provided in part by the National Science Council of the Republic of China, Grant No. NSC 88-2118-M110-001.
} 
a delayed renewal process, say $A_{d}=\left\{A_{d}(t), t \geq 0\right\}$. In particular if $H(t)=$ $\left(E\left(X_{2}\right)\right)^{-1} \int_{0}^{t} 1-F(u) d u$, then the process becomes a stationary renewal process, say $A_{s}=\left\{A_{s}(t), t \geq 0\right\}$.

When $F$ is exponential, then $A$ becomes a Poisson process. Poisson processes are related to many distributions, among them, uniform, exponential and Poisson distributions are the most important. Many interesting properties of the Poisson process are more or less based on the characteristics of these three distributions.

For example, Huang et al. (1993) prove that, given a function $G$, under mild conditions, as long as

$$
E\left(G\left(\delta_{t}\right) \mid A(t)=n\right)=E\left(G\left(X_{1}\right) \mid A(t)=n\right), \quad \forall t \geq 0,
$$

holds for a single positive integer $n$, then $A$ is a Poisson process. Li et al. (1994) and Huang and $\mathrm{Su}$ (1997) prove that for some fixed $n$ and $k, k \leq n$, if

$$
E\left(S_{k}^{r} \mid A(t)=n\right)=a t^{r} \quad \text { and } \quad E\left(S_{k}^{s} \mid A(t)=n\right)=b t^{s}, \quad \forall t \geq 0,
$$

for certain pairs of $r$ and $s$, where $a$ and $b$ are independent of $t$, then $A$ has to be a Poisson process. When $A$ is a Poisson process, the fact that (1) and (2) hold are based on the so called order statistics property. More precisely, for the Poisson process $A$, given $A(t)=n, S_{1} \leq S_{2} \leq \cdots \leq S_{n}$ are distributed as the order statistics of $n$ i.i.d. random variables with the common uniform distribution on $[0, t]$. By using this property (1) and (2) can be obtained easily.

When $A$ is a Poisson process, not only $X_{n}$, but $\gamma_{t}$, independent of $t$, is also exponentially distributed. This basically is resulted from the memoryless property of the exponential distribution. Conversely, Gupta and Gupta (1986) prove that given a function $G$, under certain conditions, if $E\left(G\left(\gamma_{t}\right)\right)=c$, $\forall t \geq 0$, where $c>0$ is a constant, then $A$ is a Poisson process. Huang et al. (1993) consider an arbitrarily delayed renewal process $A_{d}$, and let $\gamma_{t}^{d}$ denoted the residual life at $t$, they claim that for some fixed positive integer $n$, if $E\left(G\left(\gamma_{t}^{d}\right) \mid A_{d}(t)=n\right)$ is independent of $t$, then $A_{d}$ is an arbitrarily delayed Poisson process, which means the distribution of $X_{1}$ can be arbitrary, while $X_{1}, X_{2}, \ldots$ are i.i.d. exponentially distributed. If $\operatorname{Var}\left(\gamma_{t}\right)$ is constant and $F$ is assumed to be continuous, Huang and $\mathrm{Li}$ (1993) prove that $F$ is the exponential distribution function.

On the other hand, a Poisson process also has the superposition property, which says that the superposition of independent Poisson processes is still a Poisson process. Conversely, if $A$ is a Poisson process, independent thinned processes will be obtained through thinning operations on $A$. This property is a continuous time analog of the following characteristic of Poisson distribution: Assume $N$ i.i.d. multinomial trials with $k$ cells are conducted resulting into $Z_{1}, Z_{2}, \ldots, Z_{k}$ as the numbers of various types of "successes" corresponding to the $k$ cells, that is $Z_{1}+Z_{2}+\cdots+Z_{k}=N$, then the mutual independence for any $Z_{i}, Z_{j}, 1 \leq i<j \leq n$, forces $N$ to be Poisson distributed (see Moran (1952) and Patil and Seshadri (1964)). Based on the above Poisson characteristic, Chandramohan et al. (1985), Chandramohan and Liang (1985) and Huang (1989) prove that the existence of a pair of uncorrelated thinned processes implies the renewal process is Poisson. As a byproduct, Chandramohan et al. (1985) prove that for a stationary renewal process $A_{s}$,

$$
\operatorname{Var}\left(A_{s}(t)\right)=E\left(A_{s}(t)\right), \quad \forall t \geq 0,
$$


if and only if $A_{s}$ is a Poisson process. A similar result for an (ordinary) renewal process is given by Chen (1994). Note that mean and variance are equal is also an interesting property enjoyed by the Poisson random variable.

In this paper based on the above exponential and Poisson characteristics of the Poisson process, we present some characterizations of the Poisson process as a renewal process.

\section{Characterizations related to the exponential characteristic}

Let $A$ be a renewal process with $\mu_{1}=E\left(X_{1}\right)<\infty$ and $\mu_{2}=E\left(X_{1}^{2}\right)<\infty$. Huang and $\mathrm{Li}(1993)$ prove that when $F$ is continuous, $\operatorname{Var}\left(\gamma_{t}\right)=$ constant implies that the process $A$ is Poisson. Obviously, when $A$ is a Poisson process, it is also true that $\operatorname{Var}\left(\gamma_{t}\right)=E^{2}\left(\gamma_{t}\right), \forall t \geq 0$. Conversely we have the following result.

Theorem 1. Let $F$ be an absolutely continuous distribution function with density function $F^{\prime}$. Assume

$$
\operatorname{Var}\left(\gamma_{t}\right)=E^{2}\left(\gamma_{t}\right), \quad \forall t \geq 0 .
$$

Then $A$ is a Poisson process.

Proof. The following arguments are very similar to those of Theorem 1 of Huang and $\mathrm{Li}$ (1993). First from the assumption we have

$$
E\left(\gamma_{t}^{2}\right)=2 E^{2}\left(\gamma_{t}\right), \quad \forall t \geq 0 .
$$

Letting

$$
g(t)=E\left(\gamma_{t}\right)
$$

(4) implies

$$
\begin{aligned}
2 g^{2}(t)= & \int_{t}^{\infty}(x-t)^{2} d F(x)+\int_{0}^{t} E\left(\gamma_{t-x}^{2}\right) d F(x) \\
= & \int_{t}^{\infty}(x-t)^{2} d F(x)+2 \int_{0}^{t} g^{2}(t-x) d F(x) \\
= & \int_{0}^{\infty}\left(x^{2}-2 x t+t^{2}\right) d F(x)-\int_{0}^{t}(t-x)^{2} d F(x) \\
& +2 \int_{0}^{t} g^{2}(t-x) d F(x) .
\end{aligned}
$$

Taking the Laplace transforms with respect to $t$, gives

$$
2 L\left(g^{2}(t)\right)=\frac{\mu_{2}}{\theta}-\frac{2 \mu_{1}}{\theta^{2}}+\frac{2}{\theta^{3}}-\frac{2}{\theta^{3}} \phi(\theta)+2 L\left(g^{2}(t)\right) \phi(\theta),
$$


where

$$
\phi(\theta)=\int_{0}^{\infty} e^{-\theta t} d F(t),
$$

and

$$
L(q(t))=\int_{0}^{\infty} e^{-\theta t} q(t) d t
$$

denotes the Laplace transform of the function $q(t)$. On the other hand, from

$$
E\left(\gamma_{t}\right)=\int_{t}^{\infty}(x-t) d F(x)+\int_{0}^{t} E\left(\gamma_{t-x}\right) d F(x),
$$

we obtain

$$
L(g(t))=\frac{\mu_{1}}{\theta}-\frac{1}{\theta^{2}}+\frac{1}{\theta^{2}} \phi(\theta)+L(g(t)) \phi(\theta) .
$$

Now (5) and (6) can be rewritten as

$$
\frac{\mu_{2}}{\theta}-\frac{2 \mu_{1}}{\theta^{2}}=2\left(L\left(g^{2}(t)\right)-\frac{1}{\theta^{3}}\right)(1-\phi(\theta)),
$$

and

$$
\frac{\mu_{1}}{\theta}=\left(L(g(t))+\frac{1}{\theta^{2}}\right)(1-\phi(\theta)),
$$

respectively. Comparing (7) and (8) yields

$$
\frac{\mu_{2}}{\mu_{1}}-\frac{2}{\theta}=2\left(L\left(g^{2}(t)\right)-\frac{1}{\theta^{3}}\right) /\left(L(g(t))+\frac{1}{\theta^{2}}\right),
$$

which is equivalent to

$$
2 L\left(g^{2}(t)\right)=\frac{\mu_{2}}{\mu_{1}} L(g(t))+\frac{\mu_{2}}{\mu_{1}} \frac{1}{\theta^{2}}-\frac{2}{\theta} L(g(t)) .
$$

By the Uniqueness Theorem, (9) implies

$$
2 g^{2}(t)=\frac{\mu_{2}}{\mu_{1}} g(t)+\frac{\mu_{2}}{\mu_{1}} t-2 \int_{0}^{t} g(u) d u, \quad \forall t \geq 0 .
$$

The assumption that $F$ is differentiable implies $g$ is also differentiable. Hence, taking the derivatives of both sides of (10) with respect to $t$, it follows

$$
\left(4 g(t)-\frac{\mu_{2}}{\mu_{1}}\right) g^{\prime}(t)=\frac{\mu_{2}}{\mu_{1}}-2 g(t), \quad \forall t \geq 0 .
$$


Now let $t_{0}=\max \left\{t \mid t \geq 0,2 g(s)=\mu_{2} / \mu_{1}, s \leq t\right\}$, which is well defined as from (4) $2 g(0)=\mu_{2} / \mu_{1}$. Assume $t_{0}<\infty$, then $\mu_{2} / \mu_{1}=2 g\left(t_{0}\right)$. There exists a $t_{1}>t_{0}$, which is sufficiently close to $t_{0}$, such that $g^{\prime}\left(t_{1}\right) \neq 0$, and $g^{\prime}(t)$ has the same sign as $g^{\prime}\left(t_{1}\right), \forall t \in\left(t_{0}, t_{1}\right)$, while

$$
4 g\left(t_{1}\right)-\frac{\mu_{2}}{\mu_{1}}=2\left(2 g\left(t_{1}\right)-\frac{\mu_{2}}{\mu_{1}}\right)+\frac{\mu_{2}}{\mu_{1}}>0 .
$$

If $g^{\prime}\left(t_{1}\right)>0$, then $\mu_{2} / \mu_{1}-2 g\left(t_{1}\right)<0$; if $g^{\prime}\left(t_{1}\right)<0$, then $\mu_{2} / \mu_{1}-2 g\left(t_{1}\right)>0$, which both contradicts (11). Therefore $t_{0}=\infty$ and consequently

$$
g(t)=E\left(\gamma_{t}\right)=\mu_{2} /\left(2 \mu_{1}\right), \quad \forall t \geq 0,
$$

which in turn implies $F$ is exponential. Then

Next, for a stationary renewal process $A_{s}$, let $\gamma_{t}^{s}$ be the residual life at $t$.

$$
\operatorname{Var}\left(\gamma_{t}^{s}\right)=E^{2}\left(\gamma_{t}^{s}\right), \quad \forall t \geq 0
$$

can only imply

$$
2 \eta_{1} \eta_{3}=3 \eta_{2}^{2}
$$

where $\eta_{1}=E\left(X_{2}\right), \eta_{2}=E\left(X_{2}^{2}\right)$ and $\eta_{3}=E\left(X_{2}^{3}\right)$ (the derivation is left to the reader). Examples of the common distribution $F$ which satisfy (12) and are not exponential can be found easily. Note that if $F$ is assumed to be gamma distributed, then (12) implies $F$ is exponential.

\section{Characterizations related to the Poisson characteristic}

As mentioned in Section 1, for a renewal process $A$, the equality of $E(A(t))$ and $\operatorname{Var}(A(t))$ for every $t \geq 0$ implies that $A$ is a Poisson process. Similar result holds for a stationary renewal process $A_{s}$. In this section we will generalize these results. Let $M(t)=E(A(t)), M_{s}(t)=E\left(A_{s}(t)\right)$ and $\operatorname{supp}(G)$ denote the support of the function $G$.

Theorem 2. Let $E\left(X_{1}\right)<\infty, M(t)$ be continuous with $\inf \operatorname{supp}(M)=0$. Also assume $M_{+}^{\prime}(0)$ exists and that

$$
\operatorname{Var}(A(t))=b M(t), \quad \forall t \geq 0,
$$

where $b$ is a constant. Then $b=1$ and $F$ is exponential.

Proof. First we have the well-known identity

$$
E\left(\left(\begin{array}{c}
A(t) \\
k
\end{array}\right)\right)=k ! M_{k}(t), \quad \forall k \geq 2,
$$


where $M_{k}$ is the $k$-fold convolution of $M$ with itself. Hence (13) implies

$$
M_{2}(t)-\frac{b-1}{2} M(t)-\frac{1}{2} M^{2}(t)=0, \quad \forall t \geq 0,
$$

which is equivalent to

$$
\int_{0}^{t}\left(M(t-x)+M(x)-M(t)-\frac{b-1}{2}\right) d M(x)=0, \quad \forall t \geq 0 .
$$

Using Proposition 2.2 of Lau and Rao (1990), this in turn implies there exists a $\xi \in(0, t)$, such that

$$
M(t-\xi)+M(\xi)-M(t)-\frac{b-1}{2}=0, \quad \forall t \geq 0 .
$$

Since $M(0)=0$, by letting $t$ tend to 0 in the above equation, it follows $b=1$. The rest of the proof is the same as Theorem 3 of Chen (1994).

The next theorem is for the stationary renewal process. Note that it is implicitly assumed that the common interarrival time distribution function $F$ is not periodic for a stationary renewal process.

Theorem 3. Let

$$
\operatorname{Var}\left(A_{s}(t)\right)=b E\left(A_{s}(t)\right), \quad \forall t \geq 0,
$$

where $b$ is a constant. Then $b=1$ and $\left\{A_{s}(t), t \geq 0\right\}$ is a Poisson process.

Proof. Again for every positive integer $k$, by the identity

$$
E\left(A_{s}(t)\left(A_{s}(t)-1\right) \cdots\left(A_{s}(t)-k+1\right)\right)=k ! M_{s}(t) * M_{k-1}(t), \quad \forall t \geq 0,
$$

see Lemma 2.1 of Chandramohan et al. (1985), we have

$$
E\left(A_{s}(t)\left(A_{s}(t)-1\right)\right)=\frac{2}{\eta_{1}} \int_{0}^{t} M(t-x) d x=\frac{2}{\eta_{1}} \int_{0}^{t} M(x) d x,
$$

where again $\eta_{1}=E\left(X_{2}\right)$. Therefore (15) implies

$$
(b-1) \frac{t}{\eta_{1}}=\frac{2}{\eta_{1}} \int_{0}^{t} M(x) d x-\frac{t^{2}}{\eta_{1}^{2}} .
$$

Taking the derivatives of both sides with respect to $t$, then letting $t=0$, we obtain $b=1$ and $M(t)=t / \eta_{1}$ follows. Consequently $F$ is exponential as required.

Now let $\left\{\xi_{n}, b \geq 1\right\}$ be a sequence of i.i.d. random variables with $P\left(\xi_{n}=1\right)=p=1-P\left(\xi_{n}=0\right)$, where $0<p<1$. Let $A_{1}=\left\{A_{1}(t), t \geq 0\right\}$ and $A_{2}=\left\{A_{2}(t), t \geq 0\right\}$ be the two thinned processes obtained by thinning the 
renewal process $A$ through $\left\{\xi_{n}, n \geq 1\right\}$. More precisely, let the $n$-th point of $A$ be assigned to the process $A_{1}$ or $A_{2}$, respectively, according to $\xi_{n}=1$ or 0 . Obviously, $E\left(A_{1}(t)\right)=p(1-p)^{-1} E\left(A_{2}(t)\right)$, i.e. $E\left(A_{1}(t)\right)$ is proportional to $E\left(A_{2}(t)\right), \forall t \geq 0$, for every renewal process $A$. Yet given that $\operatorname{Var}\left(A_{1}(t)\right)$ is proportional to $\operatorname{Var}\left(A_{2}(t)\right), \forall t \geq 0$, will imply $A$ is Poisson. We state and prove the consequence of Theorem 2 in the following.

\section{Corollary 1. If}

$$
\operatorname{Var}\left(A_{1}(t)\right)=c \operatorname{Var}\left(A_{2}(t)\right), \quad \forall t \geq 0,
$$

where $c$ is a constant, then $A$ is a Poisson process and $c=p(1-p)^{-1}$.

Proof. First it is easy to obtain

$$
\begin{aligned}
& \operatorname{Var}\left(A_{1}(t)\right)=p(1-p) E(A(t))+p^{2} E\left(A^{2}(t)\right)-p^{2} E^{2}(A(t)), \\
& \operatorname{Var}\left(A_{2}(t)\right)=p(1-p) E(A(t))+(1-p)^{2} E\left(A^{2}(t)\right)-(1-p)^{2} E^{2}(A(t)) .
\end{aligned}
$$

Substituting these into (17), yields

$$
\operatorname{Var}\left(A(t)=\frac{p(1-p)(1-c)}{c(1-p)^{2}-p^{2}} E(A(t)) .\right.
$$

The assertions then follow from Theorem 2 immediately.

As expected, there is a similar corollary for the case of stationary renewal process. We omit the statement of this corollary. On the other hand, Theorem 3 has the following generalization, which can be proved by using (16) and the fact that $E\left(A_{s}(t)\right)=t / \eta_{1}$.

Theorem 4. The stationary renewal process $A_{s}$ is Poisson, if and only if for a fixed $k \geq 1$,

$$
E\left(A_{s}(t)\left(A_{s}(t)-1\right) \cdots\left(A_{s}(t)-k+1\right)\right)=E^{k}\left(A_{s}(t)\right), \quad \forall t \geq 0 .
$$

Acknowledgements: The authors are grateful to the referees for many helpful comments and suggestions.

\section{References}

1. Chandramohan J, Liang LK (1985) Bernoulli, multinomial and Markov chain thinning of some point processes and some results about the superposition of dependent renewal processes. J. Appl. Prob. 22:828-835

2. Chandramohan J, Foley RD, Disney RL (1985) Thinning of point processes-covariance analysis. Adv. Appl. Prob. 17:127-146

3. Chen YH (1994) Classes of life distributions and renewal counting processes. J. Appl. Prob. 31:1110-1115

4. Gupta PL, Gupta RC (1986) A characterization of the Poisson process. J. Appl. Prob. 23:233-235 
5. Huang WJ (1989) On some characterizations of the Poisson process. J. Appl. Prob. 26:176181

6. Huang WJ, Li SH (1993) Characterizations of the Poisson process using the variance. Commun. Statist.-Theory Meth. 22(5):1371-1382

7. Huang WJ, Li SH, Su JC (1993) Some characterizations of the Poisson process and geometric renewal process. J. Appl. Prob. 30:121-130

8. Huang WJ, Su JC (1997) On a study of renewal process connected with certain conditional moments. Sankhyā Ser. A 59:28-41

9. Lau KS, Rao BLSP (1990) Characterization of the exponential distribution by the relevation transform. J. Appl. Prob. 27:726-729

10. Li SH, Huang WJ, Hunag MNL (1994) Characterizations of the Poisson process as a renewal process via two conditional moments. Ann. Inst. Stat. Math. 46:351-360

11. Moran PAP (1952) A characteristic property of the Poisson distribution. Proc. Camb. Phil. Soc. 48:206-207

12. Patil GP, Seshadri V (1964) Characterization theorems for some univariate probability distributions. J. Roy. Stat. Soc. Ser. B 26:286-292 